\title{
Noethérianité de certaines algèbres de fonctions analytiques et applications
}

\author{
par Abdelhafed Elkhadiri et Mouttaki Hlal (Kinitra)
}

\begin{abstract}
Let $M \subset \mathbb{R}^{n}$ be a real-analytic submanifold and $\mathcal{H}(M)$ the algebra of real analytic functions on $M$. If $K \subset M$ is a compact subset we consider $S_{K}=\{f \in \mathcal{H}(M) \mid$ $f(x) \neq 0$ for all $x \in K\} ; S_{K}$ is a multiplicative subset of $\mathcal{H}(M)$. Let $S_{K}^{-1} \mathcal{H}(M)$ be the localization of $\mathcal{H}(M)$ with respect to $S_{K}$. In this paper we prove, first, that $S_{K}^{-1} \mathcal{H}(M)$ is a regular ring (hence noetherian) and use this result in two situations:

1) For each open subset $\Omega \subset \mathbb{R}^{n}$, we denote by $\mathcal{O}(\Omega)$ the subalgebra of $\mathcal{H}(\Omega)$ defined as follows: $f \in \mathcal{O}(\Omega)$ if and only if for all $x \in \Omega$, the germ of $f$ at $x, f_{x}$, is algebraic on $\mathcal{H}\left(\mathbb{R}^{n}\right)$. We prove that if $\Omega$ is a bounded subanalytic subset, then $\mathcal{O}(\Omega)$ is a regular ring (hence noetherian).

2) Let $M \subset \mathbb{R}^{n}$ be a Nash submanifold and $\mathcal{N}(M)$ the ring of Nash functions on $M$; we have an injection $\mathcal{N}(M) \rightarrow \mathcal{H}(M)$. In [2] it was proved that every prime ideal $\wp$ of $\mathcal{N}(M)$ generates a prime ideal of analytic functions $\wp \mathcal{H}(M)$ if $M$ or $V(\wp)$ is compact. We use our Theorem 1 to give another proof in the situation where $V(\wp)$ is compact. Finally we show that this result holds in some particular situation where $M$ and $V(\wp)$ are not assumed to be compact.
\end{abstract}

Introduction. Soit $M \subset \mathbb{R}^{m}$ une sous-variété réelle analytique; on désigne par $\mathcal{H}(M)$ l'algèbre des fonctions analytiques sur $M$. Soit $K \subset M$ un compact et considérons $S_{K}=\{f \in \mathcal{H}(M) \mid f(x) \neq 0$ pour tout $x \in K\}$; $S_{K}$ est une partie multiplicativement stable. Dans cet article on montre que le localisé de $\mathcal{H}(M)$ par rapport à $S_{K}, S_{K}^{-1} \mathcal{H}(M)$, est un anneau régulier (donc noethérien) et on applique ce résultat aux deux situations :

1) Pour chaque ouvert $\Omega \subset \mathbb{R}^{n}$, on désigne par $\mathcal{O}(\Omega)$ la sous-algèbre de $\mathcal{H}(\Omega)$ formée des $f \in \mathcal{H}(\Omega)$ telles que, pour chaque $x \in \Omega$, le germe de $f$ en $x, f_{x}$, est algébrique sur $\mathcal{H}\left(\mathbb{R}^{n}\right)$. On montre que si $\Omega$ est un ouvert

2000 Mathematics Subject Classification: 30H05, 32B20, $14 \mathrm{P} 17$.

Key words and phrases: analytic algebra, Nash functions, subanalytic sets, regular rings.

Recherches menées dans le cadre du Programme d'Appui à la Recherche Scientifique (PARS MI 33). 
borné sous-analytique, alors $\mathcal{O}(\Omega)$ est un anneau régulier (donc noethérien). Remarquons que $\mathcal{O}(\Omega)$ contient l'anneau des fonctions de Nash sur $\Omega$.

2) Soit $M \subset \mathbb{R}^{n}$ une sous-variété de Nash; on désigne par $\mathcal{N}(M)$ l'anneau des fonctions de Nash sur $M$. On a une injection $\mathcal{N}(M) \rightarrow \mathcal{H}(M)$. La question suivante a été considérée dans [2] :

$L$ 'extension d'un idéal premier, $\wp$, de $\mathcal{N}(M)$ à $\mathcal{H}(M)$ est-il premier dans $\mathcal{H}(M)$ ?

Une réponse positive dans le cas où $M$ est compacte est donnée dans [2]. Si $M$ est non compacte mais $V(\wp)$ (lieu des zéros d'un système de générateurs de $\wp$ ) est compact, la réponse est aussi positive [2]. Nous donnons une preuve directe de ce dernier cas en utilisant le théorème 1.

On désigne par $\operatorname{Reg} V(\wp) \subset V(\wp)$ les points au voisinage desquels $V(\wp)$ est une sous-variété analytique et on pose $\operatorname{Sing} V(\wp)=V(\wp)-\operatorname{Reg} V(\wp)$. Si maintenant ni $M$ ni $V(\wp)$ n'est supposé compact, la méthode que nous avons utilisée nous permet de traiter le cas particulier suivant :

Sing $V(\wp)$ est compact et pour chaque $x \in \operatorname{Reg} V(\wp), \wp \mathcal{H}_{x}$ engendre l'idéal des germes de fonctions analytiques qui s'annulent sur le germe de $V(\wp)$ en $x$.

Ces conditions sont automatiqument vérifiées dans le cas $M=\mathbb{R}^{2}$.

1. Localisation de $\mathcal{H}(M)$. Soit $M \subset \mathbb{R}^{n}$ une sous-variété réelle analytique. On désigne par $\mathcal{H}(M)$ l'anneau des fonctions analytiques sur $M$. Pour chaque $f \in \mathcal{H}(M)$ et chaque $x \in M, f_{x}$ désigne le germe de $f$ en $x$. Soit $K \subset M$; on pose $S_{K}=\{f \in \mathcal{H}(M) \mid f(x) \neq 0$ pour tout $x \in K\}$. $S_{K}$ est une partie multiplicativement stable de $\mathcal{H}(M)$; on note $S_{K}^{-1} \mathcal{H}(M)$ le localisé de $\mathcal{H}(M)$ par rapport à $S_{K}$. On a un morphisme, $i_{S}: \mathcal{H}(M) \rightarrow S_{K}^{-1} \mathcal{H}(M)$, plat. Pour chaque $x \in M, \underline{M}_{x}$ désigne l'idéal maximal des $f \in \mathcal{H}(M)$ qui s'annulent en $x$ et $\mathcal{H}_{x}$ l'anneau des germes de fonctions analytiques en $x$.

ThÉORÈme 1. Si $K$ est un compact, alors $S_{K}^{-1} \mathcal{H}(M)$ est un anneau noethérien.

Pr e u ve. Si $\bar{I}$ est un idéal de $S_{K}^{-1} \mathcal{H}(M)$, soit $I$ un idéal de $\mathcal{H}(M)$ tel que $i_{S}(I)$ engendre $\bar{I}$. On considère le faisceau d'idéaux $\mathcal{I}$ défini comme suit :

$$
\mathcal{I}_{x}=I \mathcal{H}_{x} \quad \forall x \in M .
$$

$\mathcal{I}$ est un faisceau cohérent d'idéaux sur $M$. Puisque $K$ est compact, il existe un nombre fini d'éléments de $I, f_{1}, \ldots, f_{q}$, tels que, pour chaque $x \in K$, $\mathcal{I}_{x}=\left(f_{1, x}, \ldots, f_{q, x}\right) \mathcal{H}_{x}$.

Soit $g \in I$ et considérons le faisceau cohérent d'idéaux $\mathcal{J}$ défini par

$$
\mathcal{J}_{x}=\left(f_{1, x}, \ldots, f_{q, x}\right) \mathcal{H}_{x}: g_{x} \quad \forall x \in M .
$$


Pour chaque $x \in K$ on a $\mathcal{J}_{x}=\mathcal{H}_{x}$; d'après le théorème A de Cartan, pour chaque $x \in K$ il existe un voisinage $U$ de $x$ et $\varphi^{U} \in \Gamma(M, \mathcal{J})$ tels que $\varphi^{U}(y) \neq 0$ pour tout $y \in U$. On recouvre $K$ par un nombre fini d'ouverts $U_{1}, \ldots, U_{s}$ et on pose $\varphi=\sum_{i=1}^{s}\left(\varphi^{U_{i}}\right)^{2}$. Visiblement, $\varphi(x) \neq 0$ pour tout $x \in K$ et $\varphi \in \Gamma(M, \mathcal{J})$. Soit $\mathcal{M}$ le faisceau cohérent d'idéaux défini par

$$
\mathcal{M}_{x}=\left(\varphi_{x} g_{x}, f_{1, x}, \ldots, f_{q, x}\right) \mathcal{H} x \quad \forall x \in M
$$

Il est clair que pour chaque $x \in M, \mathcal{M}_{x}$ est engendré par les germes en $x$ de $f_{1}, \ldots, f_{q}$. Le théorème $\mathrm{B}$ de Cartan entraîne alors que $\Gamma(M, \mathcal{M})$ est engendré sur $\Gamma(M, \mathcal{H})=\mathcal{H}(M)$ par $f_{1}, \ldots, f_{q}$. Comme $\varphi g \in \Gamma(M, \mathcal{M})$, on conclut alors que $\varphi g=\sum_{j=1}^{q} \lambda_{j} f_{j}, \lambda_{j} \in \mathcal{H}(M), j=1, \ldots, q$. Puisque $\varphi \in S_{K}$ on en déduit que $\bar{I}$ est engendré par $\left(f_{1}, \ldots, f_{q}\right)$.

Corollaire 1. Pour chaque $x \in M$, le localisé de $\mathcal{H}(M)$ par rapport à l'idéal $\underline{M}_{x}, \mathcal{H}(M)_{\underline{M}_{x}}$, est un anneau noethérien.

Preuve. On suppose que $M$ est connexe et que $\operatorname{dim} M=m$. Soit $x \in M$; il existe $u=\left(u_{1}, \ldots, u_{m}\right)$ éléments de $\mathcal{H}(M)$ qui forment un système de coordonnées analytiques en $x$. On a les injections suivantes :

$$
\mathbb{R}\left[u_{1}, \ldots, u_{m}\right]_{\underline{M}_{x}} \rightarrow \mathcal{H}(M)_{\underline{M}_{x}} \rightarrow \mathcal{H}_{x} .
$$

On voit alors que le complété de $\mathcal{H}(M)_{M_{x}}$ pour la topologie $\underline{M}_{x}$-adique est l'anneau des séries formelles $\mathbb{R}\left[\left[u_{1}, \ldots, u_{m}\right]\right]$. On en déduit que $\mathcal{H}(M)_{\underline{M}_{x}}$ est un anneau régulier de dimension $m$.

Proposition 1. K étant toujours compact, l'ensemble des idéaux maximaux de $S_{K}^{-1} \mathcal{H}(M)$ s'identifie à $K$.

Preuve. Soit $\widetilde{m}$ un idéal maximal de $S_{K}^{-1} \mathcal{H}(M)$; il existe alors un idéal maximal, $\underline{m}$, de $\mathcal{H}(M)$ tel que $\underline{m} \cap S_{K}=\emptyset$ et l'idéal engendré par $i_{S}(\underline{m})$ est $\widetilde{m}$. Supposons que pour chaque $x \in K$ il existe $f \in \underline{m}$ tel que $f(x) \neq 0$. On recouvre alors $K$ par un nombre fini d'ouverts $U_{1}, \ldots, U_{s}$ pour lesquels il existe $f_{i} \in \underline{m}, i=1, \ldots, s$, avec $f_{i}(y) \neq 0$ pour tout $y \in U_{i}$. On pose $\varphi=\sum_{i=1}^{s} f_{i}^{2}$; on a $\varphi \in \underline{m} \cap S_{K}$, ce qui est absurde ; donc il existe $x \in K$ tel que $\underline{m}=\underline{M}_{x}$.

Proposition 2. $S_{K}^{-1} \mathcal{H}(M)$ est un anneau régulier.

Preuve. Ceci découle de la proposition 1, du fait que $\left(S_{K}^{-1} \mathcal{H}(M)\right)_{\underline{M}_{x}}=$ $\mathcal{H}(M)_{\underline{M}_{x}}$ pour chaque $x \in K$, et du corollaire 1 .

2. Algèbres de Nash analytiques. Soit $\Omega \subset \mathbb{R}^{n}$ un ouvert; on désigne par $\mathcal{O}(\Omega)$ la sous-algèbre de $\mathcal{H}(\Omega)$ formée des $f \in \mathcal{H}(\Omega)$ telles que pour chaque $x \in \Omega$ le germe de $f$ en $x$ est algébrique sur $\mathcal{H}\left(\mathbb{R}^{n}\right)$, i.e. vérifie une équation $\sum_{i=1}^{p} a_{i} f_{x}^{i}=0$, où les $a_{i} \in \mathcal{H}\left(\mathbb{R}^{n}\right)$ sont non tous nuls. Les éléments de $\mathcal{O}(\Omega)$ seront appelés par la suite les fonctions de Nash analytiques. 
Visiblement si $f \in \mathcal{O}(\Omega)$ et $f(x) \neq 0$ pour tout $x \in \Omega$, alors $1 / f \in \mathcal{O}(\Omega)$. On désigne par $\mathcal{O}_{x}$ l'anneau des germes des fonctions de Nash analytiques en $x \in \Omega$. Il est clair que l'on a une inclusion $\mathcal{H}\left(\mathbb{R}^{n}\right)_{\underline{M}_{x}} \rightarrow \mathcal{O}_{x}$. On a, visiblement, la proposition suivante :

Proposition 3. $\mathcal{O}_{x}$ est la fermeture algébrique de $\mathcal{H}\left(\mathbb{R}^{n}\right)_{\underline{M}_{x}}$ dans son complété $\mathbb{R}\left[\left[X_{1}, \ldots, X_{n}\right]\right]$; c'est donc le hensélisé de $\mathcal{H}\left(\mathbb{R}^{n}\right)_{\underline{M}_{x}}$; en particulier $\mathcal{O}_{x}$ est un anneau régulier (donc noethérien) de dimension $n$ fidèlement plat sur $\mathcal{H}\left(\mathbb{R}^{n}\right)_{\underline{M}_{x}}$.

Corollaire 2. Si $\Omega$ est connexe, l'anneau $\mathcal{O}(\Omega)$ est intégralement clos.

Preuve. Comme $\Omega$ est connexe, les anneaux $\mathcal{O}_{x}$ et $\mathcal{H}(\Omega)$ sont canoniquement plongés dans $\mathcal{H}_{x}$ et on a $\mathcal{O}(\Omega)=\mathcal{O}_{x} \cap \mathcal{H}(\Omega)$. D'où le résultat car $\mathcal{H}(\Omega)$ est intégralement clos et de même pour $\mathcal{O}_{x}$ d'après la proposition précédente.

3. Algèbres $\Omega$-noethériennes. Une sous-algèbre $\mathcal{A}(\Omega) \subset \mathcal{H}(\Omega)$ est dite $\Omega$-noethérienne [3] si :

(i) $\mathbb{R}\left[X_{1}, \ldots, X_{n}\right] \subset \mathcal{A}(\Omega)$ et $\mathcal{A}(\Omega)$ est stable par dérivation.

(ii) $\Omega$ muni de la topologie induite par celle de $\operatorname{SM} \mathcal{A}(\Omega)$ (spectre maximal de $\mathcal{A}(\Omega)$ ) est un espace noethérien (tout point $x \in \Omega$ est identifié à l'idéal maximal, $m_{x}$, des fonctions de $\mathcal{A}(\Omega)$ qui s'annulent en $\left.x\right)$.

Pour chaque idéal $I \subset \mathcal{A}(\Omega)$ on pose $V(I)=\{x \in \Omega \mid f(x)=0$ pour tout $f \in I\}$ et on désigne par $\operatorname{Reg} V(I)$ l'ensemble des points au voisinage desquels $V(I)$ est une sous-variété; $\operatorname{Reg} V(I)$ est dense dans $V(I)$ (pour la topologie euclidienne). Si $I$ est engendré par un seul élément, $f$, on écrit $V(f)$.

Proposition 4. Soit $\mathcal{A}(\Omega) \subset \mathcal{H}(\Omega)$ une sous-algèbre stable par dérivation et contenant l'anneau des polynômes. On suppose que pour chaque $f \in$ $\mathcal{A}(\Omega), \operatorname{Reg} V(f)$ n'a qu'un nombre fini de composantes connexes. Alors $\mathcal{A}(\Omega)$ est une algèbre $\Omega$-noethérienne.

Preuve. Soit $F \subset \Omega$ un fermé pour la topologie induite par celle de $\operatorname{SM} \mathcal{A}(\Omega) ; F=V(I)$ où $I$ est un idéal de $\mathcal{A}(\Omega)$. Montrons d'abord qu'il existe un nombre fini d'éléments de $I, g_{1}, \ldots, g_{k}$, tels que $F=V\left(g_{1}, \ldots, g_{k}\right)$.

Soit $g \in I, g \neq 0$. On a $V(I) \subset V(g)$. Reg $V(g)$ n'a qu'un nombre fini de composantes connexes: $\Gamma_{1}, \ldots, \Gamma_{s_{1}}$; on pose $\mu_{1}=\max \left\{\operatorname{dim} \Gamma_{j} \mid \Gamma_{j} \not \subset V(I)\right\}$. Soient $\Gamma_{i_{1}}, \ldots, \Gamma_{i_{p}}$ les composantes connexes de Reg $V(g)$ telles que

$$
\mu_{1}=\operatorname{dim} \Gamma_{i_{j}}, \quad j=1, \ldots, p, \quad \text { et } \quad \Gamma_{i_{j}} \not \subset V(I) .
$$

Il existe alors $h_{i_{j}} \in I, h_{i_{j}} \neq 0$ sur $\Gamma_{i_{j}}, j=1, \ldots, p$. On pose $h=\sum_{j=1}^{p} h_{i_{j}}^{2}$ $\in I$; on a $V(I) \subset V(g, h) \subset V(g)$. Visiblement si $r=\max \left\{\operatorname{dim} \Lambda_{j} \mid \Lambda_{j}\right.$ est une composante connexe $\operatorname{de} \operatorname{Reg} V(g, h)$ avec $\left.\Lambda_{j} \not \subset V(I)\right\}$, alors $r<\mu_{1}$. En 
faisant la même chose pour $V(\psi)$ où $\psi=g^{2}+h^{2}$, on voit que $V(I)=V(\varphi)$ avec $\varphi \in I$.

Soit maintenant une suite de fermés, $\left(F_{i}\right)$, strictement décroissante; on a $F_{i}=V\left(f_{i}\right), f_{i} \in \mathcal{A}(\Omega)$. Associons à chaque $V\left(f_{i}\right)$ le $n+1$-uplet $\nu_{i}=$ $\left(\nu_{i, n}, \nu_{i, n-1}, \ldots, \nu_{i, 0}\right) \in \mathbb{N}^{n+1}$ tel que $\nu_{i, j}$ est le nombre des composantes connexes de $\operatorname{Reg} V\left(f_{i}\right)$ de dimension $j$. On considère sur $\mathbb{N}^{n+1}$ l'ordre lexicographique. Alors si $V\left(f_{i+1}\right) \varsubsetneqq V\left(f_{i}\right)$ on a $\nu_{i+1}<\nu_{i}$, et donc la suite $\left(F_{i}\right)$ est stationnaire.

Remarque. La condition de la proposition 4 n'est pas nécessaire; l'algèbre $\mathcal{A}(\Omega)=\mathbb{R}[x, \sin x, \cos x]$ est $\mathbb{R}$-noethérienne (même noethérienne), cependant $\operatorname{Reg} V(\sin x)=V(\sin x)$ a un nombre infini de composantes connexes.

Lemme 1. Soit $\Omega$ un ouvert borné sous-analytique. Alors l'algèbre $\mathcal{O}(\Omega)$ est $\Omega$-noethérienne et de plus $\operatorname{SMO}(\Omega)$ s'identifie à $\Omega$.

P r e u v e. On peut supposer que $\Omega$ est connexe. Il suffit, d'après la proposition 4, de montrer que pour tout $f \in \mathcal{O}(\Omega), V(f)$ est un sous-analytique de $\mathbb{R}^{n}$; en effet $\operatorname{Reg} V(f)$ sera encore sous-analytique de $\mathbb{R}^{n}$ et puisqu'il est borné, il n'aura qu'un nombre fini de composantes connexes.

On va montrer que le graphe, $G$, de $f$ est sous-analytique dans $\mathbb{R}^{n+1}$. Le problème est local en tout $a \in \partial \Omega$; on peut alors supposer que $f$ est racine d'un polynôme $P$ sans facteurs multiples à coefficients analytiques au voisinage de $\bar{\Omega}$. Soit $\Delta$ le discriminant de $P, \Delta \neq 0$; on pose $Y=\{x \in \Omega \mid$ $\Delta(x) \neq 0\}$. Visiblement $G \cap Y \times \mathbb{R}$ est une réunion de composantes connexes

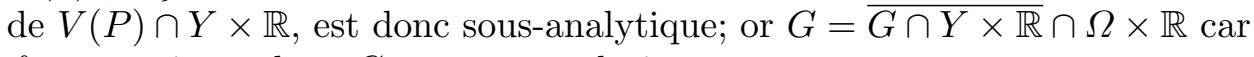
$f$ est continue, donc $G$ est sous-analytique.

Soit $\underline{m}$ un idéal maximal de $\mathcal{O}(\Omega)$; supposons que pour tout $x \in \Omega$, $\underline{m} \not \subset \underline{m}_{x}=\{f \in \mathcal{O}(\Omega) \mid f(x)=0\}$. Soit $x \in \Omega$; il existe $f_{1} \in \underline{m}$ avec $f_{1}(x) \neq 0 ; V\left(f_{1}\right)$ est non vide, sinon $f_{1}$ serait inversible dans $\mathcal{O}(\Omega)$. Soit $x_{1} \in V\left(f_{1}\right)$; comme $\underline{m} \not \subset \underline{m}_{x_{1}}$, il existe $f_{2} \in \underline{m}$ tel que $f_{2}\left(x_{1}\right) \neq 0$. On a $V\left(f_{1}, f_{2}\right) \subset V\left(f_{1}\right)$ et $V\left(f_{1}, f_{2}\right)$ est non vide, sinon $\varphi=f_{1}^{2}+f_{2}^{2} \in \underline{m}$ serait inversible dans $\mathcal{O}(\Omega)$. En continuant, on construit une suite strictement decroissante de fermés qui est infinie, ce qui est absurde. Donc il existe $x \in \Omega$ tel que $\underline{m} \subset \underline{m}_{x}$.

Dans la preuve de notre résultat, nous aurons besoin de la proposition suivante qui est une conséquence directe de [3, 6.2.1].

Proposition 5. Soit $I \subset \mathcal{A}(\Omega)$ un idéal d'une algèbre $\Omega$-noethérienne; il existe un idéal $I^{\prime} \subset I$ de type fini tel que pour tout $x \in \Omega, I^{\prime} \mathcal{H}_{x}=I \mathcal{H}_{x}$.

Proposition 6. Soient $\Omega$ un ouvert de $\mathbb{R}^{n}$ et $a \in \Omega$; alors le localisé de $\mathcal{O}(\Omega)$ par rapport à $\underline{m}_{a}, \mathcal{O}(\Omega)_{\underline{m}_{a}}$, est un anneau local régulier (donc noethérien) de dimension $n$ fidèlement plat sur $\mathcal{H}\left(\mathbb{R}^{n}\right)_{\underline{M}_{a}}$. 
Preuve. Soit $\Omega^{\prime}$ la composante connexe de $\Omega$ qui contient $a$; on a $\mathcal{O}(\Omega)_{\underline{m}_{a}}=\mathcal{O}\left(\Omega^{\prime}\right)_{\underline{m}_{a}}$. On peut alors supposer que $\Omega$ est connexe. Nous avons alors vu que $\mathcal{O}(\Omega)$ est intégralement clos; $\mathcal{O}(\Omega)_{\underline{m}_{a}}$ est donc aussi intégralement clos. On a les inclusions suivantes :

$$
\mathcal{H}\left(\mathbb{R}^{n}\right)_{\underline{M}_{a}} \rightarrow \mathcal{O}(\Omega)_{\underline{m}_{a}} \rightarrow{ }^{\mathrm{h}} \mathcal{H}\left(\mathbb{R}^{n}\right)_{\underline{M}_{a}}
$$

où ${ }^{\mathrm{h}} \mathcal{H}\left(\mathbb{R}^{n}\right)_{\underline{M}_{a}}$ est le hensélisé de $\mathcal{H}\left(\mathbb{R}^{n}\right)_{\underline{M}_{a}}$. Le résultat découle alors de $[1$, 8.7.11].

Remarquons alors que, $\Omega$ étant connexe, $\mathcal{O}(\Omega)_{\underline{m}_{a}} \rightarrow \mathcal{H}_{a}$ est fidèlement plate.

THÉORÈme 2. Soit $\Omega$ un ouvert sous-analytique borné; alors $\mathcal{O}(\Omega)$ est un anneau noethérien.

Preuve. Puisque $\Omega$ n'a qu'un nombre fini de composantes connexes, on peut supposer que $\Omega$ est connexe. Soit $I \subset \mathcal{O}(\Omega)$ un idéal; l'algèbre $\mathcal{O}(\Omega)$ étant $\Omega$-noethérienne, il existe un idéal $I^{\prime} \subset I$ de type fini tel que pour tout $x \in \Omega, I^{\prime} \mathcal{H}_{x}=I \mathcal{H}_{x}$ (proposition 5). L'injection $\mathcal{O}(\Omega)_{\underline{m}_{x}} \rightarrow \mathcal{H}_{x}$ est fidèlement plate, donc $I^{\prime} \mathcal{O}(\Omega)_{\underline{m}_{x}}=I \mathcal{O}(\Omega)_{\underline{m}_{x}}$ pour tout $x \in \Omega$. Comme $\operatorname{SMO}(\Omega)$ est identifié à $\Omega$, on en déduit, par globalisation, que $I=I^{\prime}$.

COROLlaire 3. $\Omega$ étant un ouvert sous-analytique borné, l'algèbre $\mathcal{O}(\Omega)$ est un anneau régulier de dimension $n$.

4. Problème de séparation. Soit $M \subset \mathbb{R}^{n}$ une sous-variété de Nash ; $\mathcal{N}(M)$ désigne l'anneau des fonctions de Nash sur $M$. Le problème suivant a été considéré dans [2] :

Si $\wp$ est un idéal premier de $\mathcal{N}(M)$, engendre-t-il un idéal premier dans $\mathcal{H}(M)$ ?

Une réponse positive a été donnée dans le cas où $M$ est compact [2]. Si $M$ est non compact mais $V(\wp)$ est compact, la réponse est aussi positive [2]. Nous donnons ici une preuve directe de ce dernier cas en utilisant le théorème 1 . Nous étendons le résultat au cas où $V(\wp)$ n'est pas compact mais Sing $V(\wp)$ est compact et $\wp \mathcal{H}_{x}$ engendre les germes des fonctions analytiques qui s'annulent sur le germe de $V(\wp)$ en tout $x \in \operatorname{Reg} V(\wp)$. Soit $K \subset M$ un compact; d'après le théorème $1, S_{K}^{-1} \mathcal{H}(M)$ est un anneau noethérien.

LEMME 2. L'inclusion $\varphi: \mathcal{N}(M) \rightarrow S_{K}^{-1} \mathcal{H}(M)$ est un homomorphisme régulier.

Preuve. D'après la preuve de $[4,34 . \mathrm{C}]$ il suffit de montrer que pour tout $x \in K$, l'homomorphisme $\varphi_{x}: \mathcal{N}(M)_{\underline{m}_{x}} \rightarrow \mathcal{H}(M)_{\underline{M}_{x}}$ est régulier. Soit $\left(u_{1}, \ldots, u_{m}\right)$ un système de coordonnées régulier de Nash de $M$ en $x$; on a

$$
\mathcal{N}(M)_{\underline{m}_{x}} \stackrel{\varphi_{x}}{\rightarrow} \mathcal{H}(M)_{\underline{M}_{x}} \stackrel{\psi_{x}}{\longrightarrow} \mathbb{R}\left[\left[u_{1}, \ldots, u_{m}\right]\right] .
$$


$\mathcal{N}(M)_{\underline{m}_{x}}$ est un anneau excellent, donc $\psi_{x} \circ \varphi_{x}$ est régulier. $\mathcal{H}(M)_{\underline{M}_{x}}$ est un anneau local noethérien, donc $\psi_{x}$ est fidèlement plate, d'où $\varphi_{x}$ est régulier $[4,33 . \mathrm{B}]$.

ThÉORÈme 3. Soit $\wp \subset \mathcal{N}(M)$ un idéal premier. Alors l'extension de $\wp$ à $S_{K}^{-1} \mathcal{H}(M)$ est encore un idéal premier.

Preuve. On peut supposer que $M$ est connexe. On a l'égalité $\wp=$ $\left(h_{1}, \ldots, h_{q}\right) \mathcal{N}(M)$; soient $f, g \in S_{K}^{-1} \mathcal{H}(M)$ tels que

$$
f g=\sum_{j=1}^{q} \alpha_{j} h_{j}, \quad \alpha_{j} \in S_{K}^{-1} \mathcal{H}(M), \quad j=1, \ldots, q .
$$

On pose

$$
P\left(x, Y_{1}, Y_{2}, \ldots, Y_{q+2}\right)=Y_{1} Y_{2}-\sum_{j=1}^{q} Y_{j+2} h_{j} \in \mathcal{N}(M)\left[Y_{1}, Y_{2}, \ldots, Y_{q+2}\right] .
$$

$\varphi: \mathcal{N}(M) \rightarrow S_{K}^{-1} \mathcal{H}(M)$ étant un morphisme régulier, d'après [5], $S_{K}^{-1} \mathcal{H}(M)$ est une limite inductive filtrante de $\mathcal{N}(M)$-algèbres de type fini lisses. Cela veut dire que tout homomorphisme de $\mathcal{N}(M)$-algèbres, $B \rightarrow S_{K}^{-1} \mathcal{H}(M)$, où $B$ est de type fini sur $\mathcal{N}(M)$, se factorise à travers une $\mathcal{N}(M)$-algèbre, $D$, de type fini sur $\mathcal{N}(M)$ tel que l'homomorphisme $\mathcal{N}(M) \rightarrow D$ soit régulier.

Dans notre situation, on a un $\mathcal{N}(M)$-homomorphisme donné par l'équation $(*)$ :

$$
B=\frac{\mathcal{N}(M)\left[Y_{1}, Y_{2}, \ldots, Y_{q+2}\right]}{P \mathcal{N}(M)\left[Y_{1}, Y_{2}, \ldots, Y_{q+2}\right]} \stackrel{\widetilde{\varphi}}{\rightarrow} S_{K}^{-1} \mathcal{H}(M)
$$

qui à $Y_{1}$ associe $f$, à $Y_{2}$ associe $g$ et à $Y_{j+2}$ associe $\alpha_{j}, j=1, \ldots, q$. D'après le théorème de Popescu-Spivakovsky [5], il existe alors une $\mathcal{N}(M)$-algèbre de type fini $D=\mathcal{N}(M)\left[z_{1}, \ldots, z_{m}\right] / I$ telle que l'homomorphisme $\mathcal{N}(M) \stackrel{\phi}{\rightarrow} D$ soit régulier et on a un diagramme commutatif

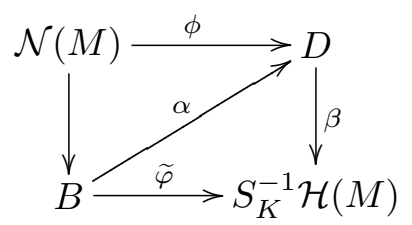

$\mathcal{N}(M) \stackrel{\phi}{\rightarrow} D$ étant un morphisme régulier, puisque $\mathcal{N}(M)$ est un anneau régulier alors $D$ est un anneau régulier, d'où $I$ est radical et donc $I=$ $\wp_{1} \cap \ldots \cap \wp_{s}, \wp_{j}$ un idéal premier de $\mathcal{N}(M)\left[z_{1}, \ldots, z_{m}\right], j=1, \ldots, s$. Tout idéal maximal, $\underline{m}$, de $\mathcal{N}(M)\left[z_{1}, \ldots, z_{m}\right]$ contenant $I$, contient un et un seul $\wp_{j}$ et

$$
\mathcal{N}(M)\left[z_{1}, \ldots, z_{m}\right]_{\underline{m}} / I=\mathcal{N}(M)\left[z_{1}, \ldots, z_{m}\right]_{\underline{m}} / \wp_{j} .
$$


$D$ est alors isomorphe au produit des $\mathcal{N}(M)\left[z_{1}, \ldots, z_{m}\right] / \wp_{j}, j=1, \ldots, s$. Comme $S_{K}^{-1} \mathcal{H}(M)$ est intègre, $\beta$ se factorise via un $\mathcal{N}(M)\left[z_{1}, \ldots, z_{m}\right] / \wp_{j}$. On peut alors supposer que $I$ est un idéal premier. Par le même argument que dans [2] on peut supposer que $I$ est réel. On pose $Z=V(I) ; Z$ est une sous-variété de Nash de $M \times \mathbb{R}^{m}$ et $D$ peut être identifié comme un sous-anneau de l'anneau des fonctions de Nash sur $Z, \mathcal{N}(Z)$.

Pour chaque $i=1, \ldots, q+2$, on pose $\alpha\left(Y_{i}\right)=H_{i} \in D$; on a

$$
H_{1} H_{2}=\sum_{j=1}^{q} H_{j+2} h_{j} \quad \text { sur } Z .
$$

On pose $\beta\left(z_{i}\right)=\sigma_{i} \in S_{K}^{-1} \mathcal{H}(M), i=1, \ldots, m$, et

$$
\sigma(x)=\left(x, \sigma_{1}(x), \ldots, \sigma_{m}(x)\right), \quad x \text { dans un voisinage de } K .
$$

Il existe alors un voisinage $W$ de $K, W \subset M$, tel que $\sigma: W \rightarrow Z$ soit analytique et une section de la projection canonique $\pi: W \times \mathbb{R}^{m} \rightarrow W$. Il existe alors une application polynomiale $P: \mathbb{R}^{n} \rightarrow \mathbb{R}^{n} \times \mathbb{R}^{m}$ qui approche $\sigma$ au voisinage de $K$. Posons $P(x)=\left(P_{0}(x), P_{1}(x), \ldots, P_{m}(x)\right), P_{0}(x) \in \mathbb{R}^{n}$.

Soit $T$ un voisinage tubulaire de $Z$ dans $W \times \mathbb{R}^{m}$ et $\varrho: T \rightarrow Z$ une rétraction de Nash compatible avec la projection canonique, i.e. $\pi \circ \varrho=\pi$ tel que $P(W) \subset T$. On pose

$$
\varrho \circ P(x)=Q(x)=\left(Q_{0}(x), Q_{1}(x), \ldots, Q_{m}(x)\right) \in Z .
$$

Comme $Q_{0}$ est proche de l'identité, quitte à rétrécir $W, Q_{0}$ est un difféomorphisme de $W$ sur $W$. Soit $Q_{0}^{-1}$ son inverse; on pose

$$
\tau(x)=\left(x, Q_{1} \circ Q_{0}^{-1}(x), \ldots, Q_{m} \circ Q_{0}^{-1}(x)\right) .
$$

Alors $\tau$ est une section de Nash de $\pi: Z \rightarrow W$, proche de $\sigma$. On pose $G_{i}=H_{i} \circ \tau, i=1, \ldots, q+2$. Alors $G_{i}$ est proche de $H_{i} \circ \sigma$ pour tout $i=1, \ldots, q+2$; de plus on a $f=H_{1} \circ \sigma, g=H_{2} \circ \sigma$ et $H_{j+2} \circ \sigma=\alpha_{j}$, $j=1, \ldots, q$.

On déduit de $(* *)$ que $f$ ou $g$ est limite de fonctions appartenant à $\wp \mathcal{H}(W)$; comme ce dernier est fermé dans $\mathcal{H}(W)$, on a, par exemple, $f \in$ $\wp \mathcal{H}(W)$.

Soit $\mathcal{J}$ le faisceau cohérent d'idéaux défini par $\mathcal{J}_{x}=\left(h_{1}, \ldots, h_{q}\right) \mathcal{H}_{x}: f_{x}$ pour tout $x \in M$. Pour chaque $x \in K, \mathcal{J}_{x}=\mathcal{H}_{x}$; d'après le théorème A de Cartan, pour chaque $x_{i} \in K$ il existe un voisinage $U_{i}$ de $x_{i}$ et $\varphi_{i} \in \Gamma(M, \mathcal{J})$ tels que $\varphi_{i}(y) \neq 0$ pour tout $y \in U_{i}$. On recouvre $K$ par un nombre fini d'ouverts $U_{i}, i=1, \ldots, l$, et on pose $\varphi=\sum_{\mu=1}^{l} \varphi_{\mu}^{2}$. Alors $\varphi \in \Gamma(M, \mathcal{J})$ et $\varphi(x) \neq 0$ pour tout $x \in K$; d'après la preuve du théorème $1, \varphi f \in$ $\left(h_{1}, \ldots, h_{q}\right) \mathcal{H}(M)$, donc $f \in \wp S_{K}^{-1} \mathcal{H}(M)$. 
Corollaire 4. Soit $\wp \subset \mathcal{N}(M)$ un idéal premier tel que $K=V(\wp)$ soit compact. Alors l'extension de $\wp$ à $\mathcal{H}(M)$ est encore un idéal premier.

Preuve. On a $\wp=\left(h_{1}, \ldots, h_{q}\right) \mathcal{N}(M)$; soient $f, g \in \mathcal{H}(M)$ tels que $f g \in \wp \mathcal{H}(M)$; d'après le théorème 3 on a, par exemple, $f \in \wp S_{K}^{-1} \mathcal{H}(M)$. Soit $\mathcal{I}$ le faisceau cohérent d'idéaux défini par

$$
\mathcal{I}_{x}=\left(h_{1}, \ldots, h_{q}, f\right) \mathcal{H}_{x}, \quad \forall x \in M .
$$

Pour chaque $x \in M, h_{1}, \ldots, h_{q}$ engendrent $\mathcal{I}_{x}$ sur $\mathcal{H}_{x}$; en effet, si $x \in$ $K$, ceci est d'après le fait que $f \in \wp S_{K}^{-1} \mathcal{H}(M)$; si $x \notin K=V(\wp)$, on a $\left(h_{1}, \ldots, h_{q}\right) \mathcal{H}_{x}=\mathcal{H}_{x}$. D'après le théorème $\mathrm{B}$ de Cartan on a $\Gamma(M, \mathcal{I})=$ $\sum_{i=1}^{q} h_{i} \mathcal{H}(M)$; comme $f \in \Gamma(M, \mathcal{I})$, on en déduit que $f \in \wp \mathcal{H}(M)$.

5. Cas particulier. Soit $I \subset \mathcal{N}(M)$ un idéal. Rappelons que $\operatorname{Reg} V(I)$ désigne les points de $V(I)$ au voisinage desquels $V(I)$ est une sous-variété; $\operatorname{Reg} V(I)$ est dense dans $V(I)$ (pour la topologie euclidienne). On pose Sing $V(I)=V(I)-\operatorname{Reg} V(I)$.

Soit $\wp \subset \mathcal{N}(M)$ un idéal premier et considérons l'hypothèse suivante :

$(\mathcal{H}) \quad$ Pour tout $x \in \operatorname{Reg} V(\wp), \wp \mathcal{H}_{x}$ engendre l'idéal des germes des fonctions analytiques qui s'annulent sur le germe de $V(\wp)$ en $x$.

ThÉORÈme 4. Soit $\wp \subset \mathcal{N}(M)$ un idéal premier. Si $(\mathcal{H})$ est vérifiée et Sing $V(\wp)$ est compact, alors $\wp \mathcal{H}(M)$ est un idéal premier.

Preuve. Posons $K=\operatorname{Sing} V(\wp)$ et soient $f, g \in \mathcal{H}(M)$ telles que $f g \in$ $\wp \mathcal{H}(M)$. D'après le théorème 3 , on a, par exemple, $f \in \wp S_{K}^{-1} \mathcal{H}(M)$, i.e. il existe $\varphi \in \mathcal{H}(M)$ avec $\varphi(x) \neq 0$ pour tout $x \in K$ et $\varphi f \in \wp \mathcal{H}(M)$. Soient $\Gamma_{1}, \ldots, \Gamma_{s}$ les composantes connexes de Reg $V(\wp)$. Puisque $V(\wp)$ est connexe et $\operatorname{Reg} V(\wp)$ est dense dans $V(\wp)$, on a $\bar{\Gamma}_{j} \cap K \neq \emptyset$ pour tout $j=1, \ldots, s$.

S'il existe $a \in \Gamma_{j}$ tel que $f(a) \neq 0$, on aura $\varphi(x)=0$ pour tout $x \in \Gamma_{j}$ et comme $\bar{\Gamma}_{j} \cap K \neq \emptyset$, il existe $b \in K$ tel que $\varphi(b)=0$, ce qui est absurde; donc $\Gamma_{j} \subset V(f)$ pour tout $j$.

L'hypothèse $(\mathcal{H})$ entraîne alors que $f_{x} \in \wp \mathcal{H}_{x}$ pour tout $x \in \operatorname{Reg} V(\wp)$, comme $\varphi f \in \wp \mathcal{H}(M)$ et $\varphi(x) \neq 0$ pour tout $x \in K$, on en déduit que $f_{x} \in \wp \mathcal{H}_{x}$ pour tout $x \in M$. Puisque $\wp$ est de type fini, d'après le théorème B de Cartan, on a $f \in \wp \mathcal{H}(M)$.

Si $M=\mathbb{R}^{2}$ et $\wp \subset \mathcal{N}(M)$ est un idéal premier, Sing $V(\wp)$ est un ensemble fini et $V(\wp)$ est connexe. Pour chaque $x \in \operatorname{Reg} V(\wp)$, ht $\left(\wp \mathcal{H}_{x}\right)=1$ et comme la dimension du germe de $V(\wp)$ en $x$ est 1 , on a alors que $\wp \mathcal{H}_{x}$ est réel, d'où le corollaire :

Corollaire 5. Si $\wp \subset \mathcal{N}\left(\mathbb{R}^{2}\right)$ est un idéal premier, alors $\wp \mathcal{H}\left(\mathbb{R}^{2}\right)$ est encore premier. 


\section{Références}

[1] J. Bochnak, M. Coste et M.-F. Roy, Géométrie algébrique réelle, Ergeb. Math. Grenzgeb. 12, Springer, New York, 1987.

[2] M. Coste, J. M. Ruiz and M. Shiota, Approximation in compact Nash manifolds, Amer. J. Math. 117 (1995), 905-927.

[3] A. Elkhadiri et J.-Cl. Tougeron, Familles noethériennes de modules sur $\underline{k}[[x]]$ et applications, Bull. Sci. Math. 120 (1996), 253-292.

[4] H. Matsumura, Commutative Algebra, Benjamin, New York, 1970.

[5] D. Popescu, General Neron desingularization, Nagoya Math. J. 100 (1985), 97-126.

Département des Mathématiques

Faculté des Sciences

Université Ibn Tofail

B.P. 133

14000 Kinitra, Morocco

E-mail: kabdelhafed@hotmail.com

Reçu par la Rédaction le 10.3.2000

Révisé le 20.8.2000 